\title{
The Improved Genetic Algorithm Used to Optimize Gabor Filters for Vehicle Detection
}

\author{
Dajiang Jin ${ }^{1}$ Lan Long $^{2}$ Jiye Zhang $^{1}$ \\ ${ }^{1}$ Traction Power State Key Laboratory, Southwest Jiaotong University, Chengdu 610031, P. R. China \\ ${ }^{2}$ Department of Mathematics and Physics, Chongqing University of Science and Technology, Chongqing 400042 , \\ P. R. China
}

\begin{abstract}
In this paper we focus on using the improved Genetic Algorithm (IGA) to optimize Gabor filters for vehicle detection. The Gabor filters parameters are encoded in a chromosome and optimized using genetic operators. Considering the deficiency of the traditional crossover operator at global searching, the partheno-crossover operator, which is a new crossover operator, is proposed and used to improve GA. The objective is to build the specifically set of filters which are capable of responding stronger to vehicles than to nonvehicles. Compared with using general GA, the Gabor filter optimized by using the IGA allows better correct rate of vehicle detection.
\end{abstract}

Keywords: Vehicle detection, Gabor filter, Genetic algorithm, Support vector machine.

\section{Introduction}

In many related applications, vehicle detection is an important problem, such as self-guided vehicles [1], driver assistance systems [2], and measurement of traffic parameters [3]. The common approach to vehicle detection is using active sensors, such as millimeter wave radars and lasers [4]. Because abundance of useful visual information can be easily got from images or videos and applied to lots of related applications [5]-[6], the approach to analyze vehicles from images or videos has attracted more and more attention lately. On the other hand, a robust and effective vehicle detection based vision is very challenging due to huge within-class variations of vehicle colors, sizes, orientations, shapes, poses, etc.

Extracting a number of features is the first main step of Building a vehicle detection system under the supervised learning framework. Due to 2-D Gabor filters is similar to receptive fields of neurons in the visual cortex [7] and have optimal joint localization both in the spatial and frequency domains, the Gabor features compared to other features including PCA and wavelet are superior in application of various image analysis. Training a classifier is another main step. Support Vector Machines (SVMs) were developed from the theory of structural risk minimization. Due to it has been shown higher performance to learning linear or nonlinear decision boundaries than traditional learning on classification problems, it has obtained much success in a variety of domains, such as image processing [8], and audio classification [9]. So we choose Gabor filters and SVMs for the task of vehicle detection.

Our focus in this paper is on using the improved Genetic Algorithm to selecting filter parameters for Gabor filters optimization. By simulating the inheritance mechanism in the evolution process according to the law of "only the fittest can survive natural selection", Genetic Algorithms (GAs) are a compute method. The idea of GAs was firstly proposed by John Holland [10]. The GAs include three operations: selection, mutation and crossover, the fundamental of the three operations is probability theory. But, in GGA, the two problems existed are immature converge and no global converge. In the past twenty years, more and more scientists have been paid attention to GAs and developed it. Goldberg et al. [11], Spears W. M. [12], Mahfoud S.W. [13]-[14] worked on the niche and selection, Srinivas M. et al. [15] do some research on adaptive parameter setting, Coli M. et al. [16], Fan Li et al. [17], Mohan C.K. et al. [18] investigated crossover operator. Maojun Li et al. [19][20] proposed the Partheno-Genetic Algorithm (PGA). In this paper, considering the deficiency of the traditional crossover operator of GAs at global searching, the partheno-crossover operator, a new crossover operator, is proposed and the parthenocrossover operator, niche technology, migration strategy and adaptive mutation are used to improve GAs

\section{2D Gabor feature and support vector machines}


We adopt a 2D Gabor filter bank for feature extraction and use these features to train SVMs for classification in this paper.

\subsection{D Gabor filter}

The 2D Gabor filter $g(x, y)$ can be represented as a Gaussian function modulated by a complex sinusoidal signal and formulated as

$$
\begin{gathered}
g(x, y)=\frac{1}{2 \pi \sigma_{x} \sigma_{y}} \exp \left[-\frac{1}{2}\left(\frac{\tilde{x}^{2}}{\sigma_{x}^{2}}+\frac{\tilde{y}^{2}}{\sigma_{y}^{2}}\right)\right] \exp [2 \pi j W \widetilde{x}], \\
\left\{\begin{array}{c}
\tilde{x}=x \cos \theta+y \sin \theta \\
\tilde{y}=-x \sin \theta+y \cos \theta
\end{array}\right.
\end{gathered}
$$

where $\sigma_{x}$ and $\sigma_{y}$ are the scaling parameters of the filter and determine the effective size of the neighborhood of a pixel in which the weighted summation takes place. $\theta(\theta \in[0, \pi))$ is the orientation of the Gabor filters. $\mathrm{W}$ is the radial frequency of the sinusoid. A filter will respond stronger to a bar or an edge with a normal parallel to the orientation $\theta$ of the sinusoid. The Fourier transform of the Gabor function is as following:

$$
G(u, v)=\exp \left[-\frac{1}{2}\left(\frac{(u-W)^{2}}{\sigma_{u}^{2}}+\frac{v^{2}}{\sigma_{v}^{2}}\right)\right],
$$

where $\sigma_{u}=1 / 2 \pi \sigma x, \sigma_{v}=1 / 2 \pi \sigma y$.

\subsection{Gabor feature extraction}

Gabor feature extraction is performed by convolving $I(x, y)$ of an input image with $g(x, y)$ of a set of Gabor filters

$$
r(x, y)=\iint I(\xi, \eta) g(x-\xi, y-\eta) d \xi d \eta,
$$

but the raw responses of the Gabor filters isn't usually used directly as features. In [6], Zehang Sun et al. used moments (the mean $\mu_{i j}$, the standard deviation $\sigma_{i j}$ and the skewness $k_{i j}$, where $i$ corresponds to the $i$ th filter and $j$ corresponds to the $j$ th subwindow) derived from Gabor filter outputs on subwindows defined on subimages extracted from the whole input image as Gabor feature extraction of postprocessing. In this paper, we also adopt this method.

\subsection{SVMs}

SVMs are primarily two-class classifiers. They were developed from the theory of structural risk minimization. Kernel functions, which satisfy Mercer's condition, can be expressed as a dot product in some space [21]. When different kernels are used, SVMs can implement different learning machines. In the experiments of Zehang Sun et al. [6], the Gaussian radial basis kernel has shown higher performance then others for application of vehicle detection. It is given by

$$
k\left(x, x_{1}\right)=\exp \left(-\frac{\left\|x-x_{i}\right\|^{2}}{2 \delta^{2}}\right)
$$

\section{Improved genetic algorithm}

In this section, the partheno-crossover operator, niche technology, migration strategy and adaptive mutation will be taken to improve GAs.

\subsection{Encoding and decoding}

We use a binary encoding scheme. Every parameter of Gabor filter is represented by $m$ bits of binary system. If there are $n$ parameters, $X_{i} \in\left[a_{i}, b_{i}\right]$, and reserve $c_{i}$ decimals $(i=1, \ldots, n)$, the value of $m_{i}$ is computed by

$$
2^{m_{i}-1}<\left(b_{i}-a_{i}\right) \times 10^{c_{i}} \leq 2^{m_{i}}-1 \quad i=1, \cdots, n .
$$

So $n$ corresponding binary numbers of the parameters combine into a chromosome in order. Then the decoding formula of $X_{i}$ is given by

$$
X_{i}=a_{i}+\operatorname{dec}\left(\operatorname{bin}_{i}\right) \times \frac{b_{i}-a_{i}}{2^{m_{i}}-1} \quad i=1, \cdots, n,
$$

where $\operatorname{dec}\left(\operatorname{bin}_{i}\right)$ expresses the corresponding decimal number of the binary number of $X_{i}$.

\subsection{Initial population}

To increase the diversity of the initial population, the individuals of the initial population are uniformly generated from searching space.

\subsection{Selection}

Niche technology is used to remove redundant individuals and to select fittest individuals. In this paper, hamming distance (genetic difference) $\sigma_{l}$ and fitness distance (fitness margin) $\sigma_{2}$ are used to define radius of niche:

$$
\left\{\begin{array}{l}
\sigma_{1}=\frac{m}{2} \\
\sigma_{2}=\frac{f_{\text {max }}-f_{\text {min }}}{4 n},
\end{array}\right.
$$

where $m$ is chromosome length, $n$ is population size, $f_{\max }$ is maximum values of fitness in the population, and $f_{\min }$ is minimum values of fitness in the population. When two individuals' Hamming distance and fitness distance are less than the threshold value, they belong to the same niche. In the same niche, the individual of the largest fitness value is retained and others are eliminated. Some positions of population are vacant later. 
The migrant individual is produced through the two-step crossover operator. First, the initial immigrant individuals are produced by the parthenocrossover operator in the individual retained. All initial immigrant individuals form a population. Second, by used traditional crossover operator, the population is operated one time and a new population is produced. Vacant positions are filled by the immigrant individuals of the new population.

\subsection{Mutation}

In order to keep the excellent quality of individual gene fragments, adaptive mutation is used. Each individual's mutation probability is as following:

$$
p_{m}^{\prime}= \begin{cases}p_{m} \frac{f_{\max }-f}{f_{\max }-f_{\text {avg }}} & f>f_{\text {avg }}, \\ 3 p_{m} & \text { others }\end{cases}
$$

where $p_{m}^{\prime}$ is mutation probability adjusted, $p_{m}$ is mutation probability, $f_{\max }$ is maximum values of fitness in the population, $f_{\min }$ is minimum values of fitness in the population and $f_{\text {avg }}$ is average values of fitness in the population.

\subsection{The partheno-crossover operator proposed}

In this paper, the individuals continue to cross in population is the main reason for the immature converges, because it can make most of individuals to next of kin. So the individuals no longer cross between with each other in the same population. The parthenocrossover operator, a new crossover operator, is proposed. The partheno-crossover operator let the individual selected in the population crosses over the individual of the completely difference to it. For example, there is the individual selected in the population, 011010001001011110. The completely different individual is 100101110110100001 . If they cross to one times in the 9 th locus, two offspring are produced, 011010001110100001

and 100101110001011110 .

When they cross to $n$ times, there are $2 n$ offspring and only the fittest offspring can replace his father's position in the population. So this offspring either inherits the father's good gene fragments or have a better gene fragments. It both ensures that the population develops to excellent direction, and avoids that the population converges to a local optimal solution. It improves the algorithm's performance in local and global search. Although the parthenocrossover operator isn't a partheno-genetic because all individuals of population have spouses fixed, only one of parent is in the population. So we named it partheno-crossover operator.

\subsection{Fitness evaluation}

The fitness value of each chromosome is the performance of a SVM classifier on features extracted from the responses of the corresponding Gabor filters of the chromosome. This method is a closed-loop learning scheme, which can make the Gabor filter design more powerful.

\subsection{IGA procedure}

Using method introduced as above, the procedure of IGA is as follows. Fig. 1 is flowchart of PCGA Procedure.

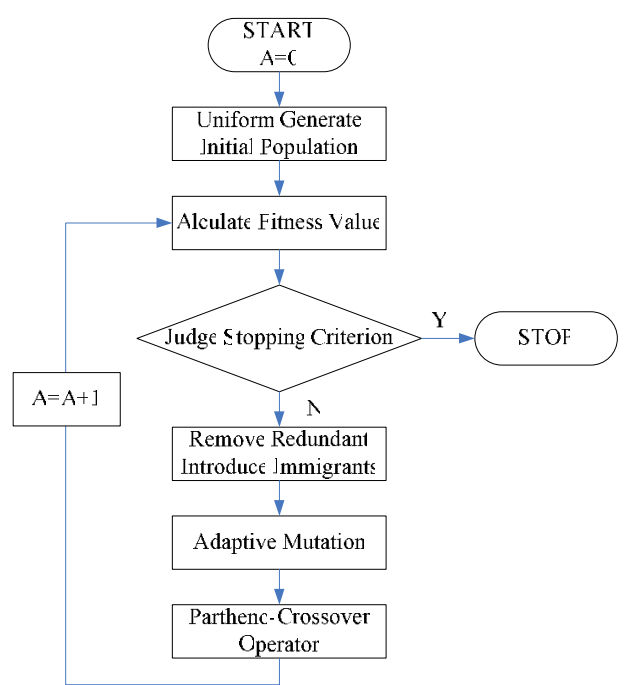

Fig. 1: Flowchart of IGA procedure.

- STEP1. Set the parameters: the maximum number of generations $g$, the population size $n$, the mutation probability $p_{m}$, crossover probability $p_{c}$, the number of generations of convergence $c$ and the number of generations A.

- SETP2. Uniform generate initial population.

- SETP3. Calculate fitness value. If the stopping criterion is satisfied, go to Step8; else continue. The stopping criterion is that $A=g$ or the best fitness keeping no change in the last $c$ generations and this fitness must also equal the best fitness found once.

- SETP4. Remove redundant individuals and select fittest individuals. Introduce immigrant individual.

- SETP5. Adaptive mutation.

- $\quad$ SETP6. Partheno-crossover operator 
- $\quad$ SETP7. $A=A+1$. Then go to SETP3.

- $\quad$ SETP8. Output the best solution and stop.

\section{Testing algorithms}

In this section, we consider the problem of vehicle detection from gray-scale images.

\subsection{Vehicle data}

The images used in our test include 100 vehicles and 100 nonvehicles images extracted manually, in which 10 vehicle and 10 nonvehicle images are training set and the others are test set, such as Fig. 2. In vehicle images, each vehicle was approximately put to the same position. All of the images were scaled to a size of $64 \times 64$.

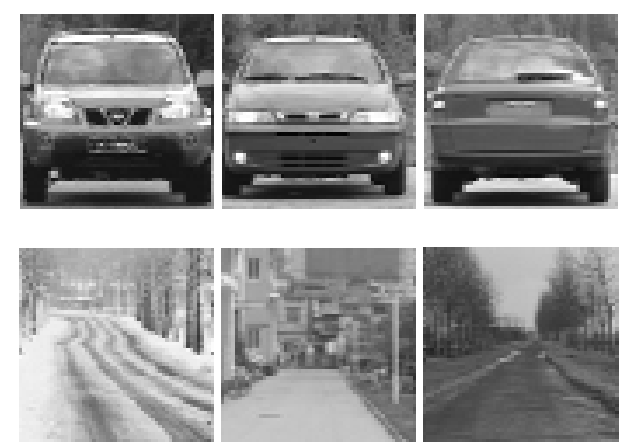

Fig. 2: The training and testing images.

\subsection{Parameters of GA}

In the tests, let the population size $n=25$, crossover probability $p_{c}=0.66$, mutation probability: $p_{m}=0.03$, the maximum number of generations $g=1000$, the number of generations of convergence $c=10$, respectively.

\subsection{Testing result}

For comparison purposes, we also used the Gabor filter bank of four scales and six orientations and EGFO (without clustering) [6] except the Gabor filter optimized by using the IGA (GFOI). And the GA of EGFO and GFOI use two methods: one with convergence judgment, the other without convergence judgment. There are the testing results in Table 1.

In the table 1 , it is shown that EGFO without convergence judgment, GFOI without convergence judgment and GFOI with convergence judgment nearly get the same correct rate. But GFOI with convergence judgment has fewer generations, and the correct rate of it is much better than that of EGFO without convergence judgment. So it indicates that the
IGA have better ability and stability of globe searching than the general GA of EGFO.

\begin{tabular}{|c|c|c|c|c|}
\hline $\begin{array}{c}\text { Correct } \\
\text { Rates (\%) }\end{array}$ & Set 1 & Set 2 & Set 3 & Average \\
\hline $\begin{array}{c}\text { the Gabor } \\
\text { filter bank } \\
\text { (4×6) }\end{array}$ & 87.78 & 85.00 & 90.56 & 87.78 \\
\hline $\begin{array}{c}\text { EGFO } \\
\text { (without } \\
\text { convergence } \\
\text { judgment) }\end{array}$ & 95.56 & 91.67 & 92.78 & 93.33 \\
\hline $\begin{array}{c}\text { EGFO } \\
\text { (with } \\
\text { convergence } \\
\text { judgment) }\end{array}$ & $55.56(97)$ & $72.22(219)$ & $16.67(45)$ & $48.15(120)$ \\
\hline $\begin{array}{c}\text { GFOI } \\
\text { (without } \\
\text { convergence } \\
\text { judgment) }\end{array}$ & 95 & 94.44 & 92.23 & 93.89 \\
\hline $\begin{array}{c}\text { GFOI } \\
\text { (with } \\
\text { convergence } \\
\text { judgment) }\end{array}$ & $93.89(473)$ & $88.89(154)$ & $91.67(277)$ & $91.46(301)$ \\
\hline \multicolumn{2}{|c|}{ Table 1: The testing results } \\
\hline
\end{tabular}

(In parentheses indicate the number of generation).

\section{Conclusion}

In this paper, the partheno-crossover operator, niche technology, migration strategy and adaptive mutation were taken to improve GAs. The IGA have better capability and stability of globe searching than the general GA of EGFO. Using the IGA to optimize Gabor filters allows the better specifically set of filters. Using the specifically set of filters, the correct rate of vehicle detection increases.

\section{Acknowledgements}

This work is supported by National Program for New Century Excellent Talents in University (No. NCET04-0889) and Youth Science Foundation of Sichuan (No. 05ZQ026-015).

\section{References}

[1] M. Bertozzi, A. Broggi and S. Castelluccio, A real-time oriented system for vehicle detection. Journal of Systems Architecture, 43(2):317-325, 1997.

[2] Z. Sun, G. Bebis and R. Miller, On-road vehicle detection: A review. IEEE Trans. Pattern Anal. Mach. Intell., 28(5):694-711, 2006. 
[3] R. Cucchiara, P. Mello and M. Piccardi, Image analysis and rulebased reasoning for a traffic monitoring. IEEE Trans. Intell. Transport. Syst., 3(1):37-47, 2002.

[4] W. Jones, Building safer cars. IEEE Spectrum, 39(1):82-85, 2002.

[5] Y. Ohta, Pattern recognition and understanding for visual information media. Proceedings of 16th International Conference, 1:536-545, Aug. 2002.

[6] Z. Sun, G. Bebis and R. Miller, On-Road Vehicle Detection Using Evolutionary Gabor Filter Optimization. IEEE Trans. on Intelligent Transportation Systems, 6(2):125-137, 2005.

[7] J. Daugman, Complete discrete 2-D Gabor transforms by neural network for image analysis and compression. IEEE Trans. Acoust. Speech Signal Process. 36:1169-1179, 1988.

[8] K. I. Kim, K. Jung and J. H. Kim, Texture-based, approach for text detection in images using support vector machines, and continuously adaptive mean shift algorithm. IEEE Trans. on Pattern Analysis and Machine Intelligence, 25(12):1631-1639, 2003.

[9] G. Guo and S. Z. Li, Content-based audio classification and retrieval by support vector machines. IEEE Trans. on Speech and Audio Processing, 14(1):209-215, 2003.

[10] J. H. Holland, Adaptation in Natural Artificial Systems [M], MIT Press, 1975.

[11] D. E. Goldberg and J. Rechardson, Genetic Algorithms with Sharing for Multimodal Optimization. Proceedings of the Second International Conference on Genetic Algorithms, pp. 69-76, 1987.

[12] W. M. Spears, Simple subpopulation schemes, Proceedings of the Third Annual Conference on Evolutionary Programming. pp. 296-307, 1994.
[13] S. W. Mahfoud, Crossover interactions among niches. Proceedings of the First IEEE Conference on Evolutionary Computation, pp. 188-193, 1994.

[14] S. W. Mahfoud, A comparison of parallel and sequential niching methods. Proceedings of the Sixthly International Conference on Genetic Algorithms, pp. 136-143, 1995.

[15] M. Srinivas and L.M. Patmaik, Adaptive Probabilities of Crossover and Mutations in genetic algorithms. IEEE Trans. on Systems, Man, and Cybernetics, 24:656-665, 1994.

[16] M. Coli, G. Gennuso and P. Palazzari, A new crossover operator for genetic algorithms. Proceedings of IEEE International Conference on Evolutionary Computation, pp. 201-206, 1996.

[17] F. Li, Q. Liu, F. Min and G. Yang, A new crossover operator based on the rough set theory for genetic algorithms. Proceedings of 2005 International Conference on Machine Learning and Cybernetics, 5:2907-2912, 2005.

[18] C. K. Mohan, Crossover operators that improve offspring fitness. Proceedings of the 1999 Congress on Evolutionary Computation, 2:1549, 1999.

[19] M. Li and T. Tong, A patiheno-genetic algorithm and analysis on its global convergence. Acta Automatic Sinica, 25:68-72, 1999.

[20] M. Li and T. Tong, An improved parthenogenetic algorithm for traveling salesman problem. Proceedings of the 4th World Congress on Intelligent Control and Automation, 4:3000-3004, 2002.

[21] V. Vapnik, The Nature of Statistical Learning Theory. Springer-Verlag, 1995. 